\title{
G-forces in endocrinology
}

\author{
Aart Jan van der Lely
}

When attending one of the major meetings in endocrinology, one cannot escape the feeling that we are dealing with increasing $\mathrm{G}$-forces. What I mean is the increasing number of sessions and presentations that address the fascinating properties of the so-called G-protein-coupled receptors (GPCRs), also known as seven-transmembrane receptors. Some of them do not use G-proteins; therefore, seven-transmembrane receptors might actually be a better description of these molecules.

GPCRs comprise a large protein family of transmembrane receptors that sense molecules outside the cell and activate signal transduction pathways inside. It is worth noting that more than $50 \%$ of the drugs in the pharmaceutical arsenal currently target GPCRs as part of their mode of action. Only a minority of the hundreds of GPCRs are characterized well enough so that we know their active ligand(s). Researchers are, however, making rapid progress in 'reuniting' families of ligands with their orphan receptors, and this process will inevitably bring along a whole stream of innovative medications.

Science is, moreover, increasing our knowledge about how GPCRs work and how they interact with their ligands. One of the most recent steps forward was presented by Holst and co-workers (Holst B et al. [2007] J Biol Chem 282: 15799-15811). In experiments with the ghrelin receptor, they observed that the relative position of a ligand in the binding pocket between the 'efficacy shift region' on the third transmembrane (TM-III) part of the receptor and the opposing aromatic cluster on TM-VI and TM-VII either leads to agonism, when the ligand is in a superficial binding mode, or leads to inverse agonism, when the ligand is in a deeper binding position. This relationship
"... rapid

progress in

'reuniting'

families of

ligands with

their orphan

receptors ... will inevitably

bring along a whole stream of innovative medications"

AJ van der Lely is an Advisory Board member of Nature Clinical Practice Endocrinology \& Metabolism.

\section{Competing interests}

The author declared he has no competing interests.

www.nature.com/clinicalpractice doi:10.1038/ncpendmet0564 between different binding modes and opposite efficacy is in accordance with the so-called Global Toggle Switch model for GPCR receptor activation that was previously discussed by the same group of researchers (Schwartz TW et al. [2006] Annu Rev Pharmacol Toxicol 46: 481-519).

The problem in understanding GPCR action is that a huge number of chemically very different agonists do not share a common binding mode or active site on their GPCRs, but nevertheless act through induction of a common molecular activation mechanism. The Global Toggle Switch model that was proposed by Schwartz et al. predicted an outward rigidbody movement of the intracellular segments, as well as an opposite, inward movement of the extracellular segments of the transmembrane helices. According to their model, what they call a vertical see-saw movement of $\mathrm{TM}-\mathrm{VI}-$ and to some degree $\mathrm{TM}-\mathrm{VII}$ - around a pivot will occur during receptor activation, which might involve the outer segment of TM-V in an as-yet-unclear fashion.

This might sound like science fiction to most of us, including me, but what it also means is that very soon agonists and antagonists can be predicted with much higher reliability; therefore, making designer molecules will be easier and, in the end, this will improve patient care. When we look, for example, at the ghrelin receptor, we will see some agonists becoming available for human studies in catabolism very soon. With improving insight about receptor structure-function relations, the speed by which these agonists and other compounds can be synthesized will increase dramatically. The $\mathrm{G}$-forces are with us and getting stronger-be aware of that! 